\title{
ON FUCHS' RELATION FOR THE LINEAR DIFFERENTIAL EQUATION WITH ALGEBRAIC COEFFICIENTS
}

\author{
By Tosiya SaIto
}

1. In the theory of linear differential equations of Fuchsian type with rational coefficients, it is well known that the sum of the roots of indicial equations of an equation of this type is equal to

$$
n(n-1)\left(\frac{m}{2}-1\right)
$$

where $n$ is the order of the equation and $m$ is the number of its singular points. The purpose of this paper is to generalize this Fuchs' relation for the case when the coefficients of the equation are algebraic.

The equation we are to investigate is

$$
\frac{d^{n} v}{d x^{n}}+p_{1} \frac{d^{n-1} v}{d x^{n-1}}+\cdots+p_{n} v=0
$$

where the coefficients $p_{1}, p_{2}, \cdots, p_{n}$ are all supposed to be one-valued and meromorphic functions on a Riemann surface $\mathfrak{F}$ of an algebraic function $y=\varphi(x)$ with genus $p$ and consisting of $r$ sheets.

We denote by

$$
\begin{aligned}
& \mathfrak{q}_{1}=\left(\alpha_{1}, \beta_{1}\right), \cdots, \quad \mathfrak{q}_{s}=\left(\alpha_{s}, \beta_{s}\right) ; \\
& \mathfrak{r}_{1}=\left(\infty, \gamma_{1}\right), \cdots, \quad \mathfrak{r}_{r}=\left(\infty, \gamma_{r}\right) ; \\
& \mathfrak{p}_{1}=\left(a_{1}, b_{1}\right), \cdots, \quad \mathfrak{p}_{m}=\left(a_{m}, b_{m}\right)
\end{aligned}
$$

the branch points of $\varphi(x)$, the points at infinity on $\mathfrak{F}$, and the singular points of the equation (1) respectively, where the notation $(\alpha, \beta)$ stands for the point of $\mathfrak{F}$ such that $x=\alpha, y=\beta$. For simplicity's sake, we assume that

$$
\begin{array}{ll}
\mathfrak{p}_{\jmath} \neq \mathfrak{q}_{k}, & \text { for } j=1, \cdots, m \text { and } k=1, \cdots, s, \\
\mathfrak{p}_{\jmath} \neq \mathfrak{r}_{k}, & \text { for } j=1, \cdots, m \text { and } k=1, \cdots, r, \\
\mathfrak{q}_{\jmath} \neq \mathfrak{r}_{k}, & \text { for } j=1, \cdots, s \text { and } k=1, \cdots, r .
\end{array}
$$

2. We suppose that the equation (1) is of Fuchsian type. To derive the generalized Fuchs' relation for this equation, we must investigate the behaviour of the coefficient $p_{1}$ on $\mathfrak{F}$.

First, $p_{1}$ must have a pole of order at most 1 at $\mathfrak{p}_{1}, \cdots, \mathfrak{p}_{m}$. Accordingly it is expanded, in the neighbourhood of each $\mathfrak{p}_{\jmath}$, in the form

Received March 24, 1958. 


$$
p_{1}=\left(x-a_{j}\right)^{-1}\left[A_{j 0}+A_{j 1}\left(x-a_{j}\right)+\cdots\right] .
$$

Next, we consider the equation (1) in the neighbourhood of $\mathfrak{q}_{\jmath}$. If it is a branch point of order $m_{\jmath}-1\left(m_{\jmath}>1\right)$, we can take

$$
\tau=\left(x-\alpha_{j}\right)^{1 / m_{j}}
$$

as a local uniformizing variable at $\mathfrak{q}_{\jmath}$. Then, as can easily be shown by induction, we have

$$
\begin{aligned}
& \frac{d^{k} v}{d x^{k}}=\left(\frac{1}{m_{\jmath}}\right)^{k} \tau^{k-k m_{\jmath}} \frac{d^{k} v}{d \tau^{k}}+\frac{k(k-1)}{2}\left(\frac{1}{m_{\jmath}}\right)^{k-1}\left(\frac{1}{m_{\jmath}}-1\right) \tau^{k-1-k m_{\jmath}} \frac{d^{k-1} v}{d \tau^{k-1}}+\cdots, \\
& k=1,2, \cdots \text {. }
\end{aligned}
$$

Substituting these relations in the left-hand side of the equation (1), we obtain

$$
\begin{aligned}
& \frac{d^{n} v}{d x^{n}}+p_{1} \frac{d^{n-1} v}{d x^{n-1}}+\cdots+p_{n} v \\
= & \left(\frac{1}{m_{\jmath}}\right)^{n} \tau^{n-n m_{\jmath}} \frac{d^{n} v}{d \tau^{n}}+\frac{n(n-1)}{2}\left(\frac{1}{m_{\jmath}}\right)^{n-1}\left(\frac{1}{m_{\jmath}}-1\right) \tau^{n-1-n m_{\jmath}} \frac{d^{n-1} v}{d \tau^{n-1}}+\cdots \\
& +p_{1} \cdot\left(\frac{1}{m_{\jmath}}\right)^{n-1} \tau^{n-1-(n-1) m_{\jmath}} \frac{d^{n-1} v}{d \tau^{n-1}}+\cdots
\end{aligned}
$$

Therefore, in this new variable $\tau$, the equation (1) will be written as

$$
\frac{d^{n} v}{d \tau^{n}}+Q_{1} \frac{d^{n-1} v}{d \tau^{n-1}}+\cdots+Q_{n} v=0
$$

where

$$
Q_{1}=m_{j} \tau^{-1}\left[\begin{array}{c}
n(n-1) \\
2
\end{array}\left(\frac{1}{m_{j}}-1\right)+\tau^{m_{j}} p_{1}\right]
$$

$\mathfrak{q}_{\text {J }}$ being a regular point of the equation (1), $Q_{1}$ must be regular at $\tau=0$. Hence we must have

$$
p_{1}=\tau^{-m_{j}}\left[-\frac{n(n-1)}{2}\left(\frac{1}{m_{j}}-1\right)+B_{j 1} \tau+\cdots\right] .
$$

Consequently, in the neighbourhood of each $\mathfrak{q}_{\jmath}, p_{1}$ is expanded in the form

where

$$
p_{1}=\left(x-\alpha_{j}\right)^{-1}\left[B_{j 0}+B_{j 1}\left(x-\alpha_{j}\right)^{1 / m_{\jmath}}+\cdots\right],
$$

$$
B_{j 0}=-\frac{n(n-1)}{2}\left(\frac{1}{m_{j}}-1\right) .
$$

Finally, we must determine the behaviour of $p_{1}$ at $\mathfrak{q}_{\jmath}$. For that purpose, however, it suffices to replace $m_{3}$ by -1 and $\tau=\left(x-\alpha_{j}\right)^{1 / m}$, by $\tau=x^{-1}$ in above discussions. Thus, in the neighbourhood of each $\mathfrak{r}_{\jmath}, p_{1}$ is expanded in the form

$$
p_{1}=x^{-1}\left[C_{j 0}+C_{j 1} x^{-1}+\cdots\right] \text {, }
$$


where

$$
C_{j 0}=n(n-1) .
$$

Evidently $p_{1}$ should be regular elsewhere on $\mathfrak{F}$.

3. Let

$$
\begin{aligned}
& \Gamma_{1}, \quad \Gamma_{2}, \cdots, \Gamma_{m} ; \\
& \Gamma_{1}{ }^{\prime}, \Gamma_{2}{ }^{\prime}, \cdots, \Gamma_{s}{ }^{\prime} ; \\
& \Gamma_{1}{ }^{\prime \prime}, \Gamma_{2}{ }^{\prime \prime}, \cdots, \Gamma_{r}{ }^{\prime \prime}
\end{aligned}
$$

be small circuits around

$$
\begin{aligned}
& \mathfrak{p}_{1}, \mathfrak{p}_{2}, \cdots, \mathfrak{p}_{m} ; \\
& \mathfrak{q}_{1}, \mathfrak{q}_{2}, \cdots, \mathfrak{q}_{s} ; \\
& \mathfrak{x}_{1}, \mathfrak{x}_{2}, \cdots, \mathfrak{x}_{r},
\end{aligned}
$$

respectively. Evidently we have

$$
\int_{\Gamma_{\jmath}} p_{1} d x=\int_{\Gamma_{\jmath}}\left(x-a_{j}\right)^{-1}\left[A_{j 0}+A_{j 1}\left(x-a_{j}\right)+\cdots\right] d x=2 \pi i A_{j 0},
$$

where the integration is to be carried out counterclockwise. Also, by putting $\tau=\left(x-\alpha_{j}\right)^{1 / m_{\jmath}}$, we have

$$
\begin{aligned}
\int_{\Gamma^{\prime} j^{\prime}} p_{1} d x & =\int_{\Gamma^{\prime} j^{\prime}}\left(x-\alpha_{j}\right)^{-1}\left[B_{j 0}+B_{j 1}\left(x-\alpha_{j}\right)^{1 / m_{\jmath}}+\cdots\right] d x \\
& =\int_{\Gamma^{\prime} j^{\prime}} \tau^{-m_{j}}\left[B_{j 0}+B_{1} \tau+\cdots\right] m_{j} \tau^{m_{j}-1} d \tau=2 \pi i m_{\jmath} B_{j 0} .
\end{aligned}
$$

Similarly, putting $\tau=x^{-1}$, we have

$$
\begin{aligned}
\int_{\Gamma^{\prime} j^{\prime \prime}} p_{1} d x & =\int_{\Gamma j^{\prime \prime}} x^{-1}\left[C_{j 0}+C_{j 1} x^{-1}+\cdots\right] d x \\
& =\int_{\Gamma j^{\prime \prime}} \tau\left[C_{j 0}+C_{j 1} \tau+\cdots\right](-1) \tau^{-2} d \tau=-2 \pi i C_{j 0} .
\end{aligned}
$$

Since $p_{1}$ is one-valued and the differential $p_{1} d x$ is regular elsewhere, we have

$$
\sum_{j=1}^{m} \int_{\Gamma_{j}} p_{1} d x+\sum_{j=1}^{s} \int_{\Gamma_{j^{\prime}}} p_{1} d x+\sum_{j=1}^{r} \int_{\Gamma^{\prime} j^{\prime \prime}} p_{1} d x=0
$$

whence follows the relation

$$
\sum_{j=1}^{m} A_{j 0}+\sum_{j=1}^{s} m_{\jmath} B_{j 0}-\sum_{\jmath=1}^{r} C_{j 0}=0 .
$$

4. Fuchs' relation for the equation (1) can be derived immediately from relation (5).

An indicial eqnation (1) at $p_{j}$ is given by 


$$
\lambda(\lambda-1) \cdots(\lambda-n+1)+A_{j 0} \cdot \lambda(\lambda-1) \cdots(\lambda-n+2)+\cdots=0,
$$

which can be rewritten in the form

$$
\lambda^{n}+\left[-1-2-\cdots-(n-1)+A_{j 0}\right] \lambda^{n-1}+\cdots=0 .
$$

Therefore, if $\lambda_{j 1}, \cdots, \lambda_{j n}$ are its roots, we have

$$
\begin{aligned}
\sum_{k=1}^{n} \lambda_{j k} & =1+2+\cdots+(n-1)-A_{j 0} \\
& =\frac{n(n-1)}{2}-A_{j 0} .
\end{aligned}
$$

From this follows that

$$
\sum_{j=1}^{m} \sum_{k=1}^{n} \lambda_{j k}=\frac{m n(n-1)}{2}-\sum_{j=1}^{m} A_{j 0} .
$$

Now, from (3), (4) and (5), we get

$$
\begin{aligned}
\sum_{j=1}^{m} A_{j 0} & =-\sum_{j=1}^{s} m_{j} B_{j 0}+\sum_{j=1}^{r} C_{j 0} \\
& =-\frac{n(n-1)}{2} \sum_{j=1}^{s}\left(m_{\jmath}-1\right)+r n(n-1) .
\end{aligned}
$$

Since a well-known formula of Hurwitz

$$
\sum_{j=1}^{s}\left(m_{J}-1\right)=2(r+p-1)
$$

holds, we have

$$
\begin{aligned}
\sum_{j=1}^{m} A_{j 0} & =-n(n-1)(r+p-1)+r n(n-1) \\
& =-n(n-1)(p-1) .
\end{aligned}
$$

Thus will follow the generalized Fuchs' relation:

$$
\sum_{j=1}^{m} \sum_{k=1}^{n} \lambda_{j k}=n(n-1)\left(\frac{m}{2}+p-1\right) .
$$

DEPARTMENT OF MATHEMATICS,

Tokyo Institute of TeChNology. 